\title{
Symbiosis between neuroscience and computing
}

Francisco Cedron, Sara Alvarez-Gonzalez, Ana Belen Porto-Pazos

This paper presents how two apparently distinct branches, neuroscience and artificial intelligence, can collaborate in a symbiosis that allows them to join forces for new discoveries. Researchers specialising in the field of neuroscience can put forward conjectures and hypotheses that they have not been able to test for various reasons (high cost of testing, highly specialised simulations that programmes such as Neuron do not allow, etc.). All these questions posed by neuroscientists can be implemented by artificial intelligence experts within the connectionist branch. This allows new paradigms to appear in the artificial intelligence branch, and in the neuroscience branch to have a clearer idea of which hypothesis makes more sense in information processing. An example of this symbiosis has been the study of the tripartite synapse between neuron and astrocytes. 